\title{
Developments in RNA interference and genetic transformation to define gene function in parasitic helminths
}

\author{
David P. Knox
}

(Received 4 Fanuary 2012; revised 5 Fanuary 2012; accepted 5 Fanuary 2012)

\begin{abstract}
Advances in DNA sequencing technologies have led to the rapid accumulation of gene sequence data with whole genome sequences in draft form available for a number of important helminth parasites of animals and plants. There is an urgent requirement to establish reverse genetics tools to define the function of individual genes to enhance our knowledge of parasitism and to identify key parasite genes and their products which can provide the basis for novel control procedures be these new chemical interventions or vaccines. RNA interference (RNAi) is a reverse genetics technique which permits the gene-specific degradation of mRNA by the introduction of complementary double-stranded RNA (dsRNA). This has been achieved by a variety of approaches including soaking, electroporation or, in the case of Caenorhabditis elegans, by microinjection. The discovery that RNAi could be induced by simply soaking $C$. elegans the worm in dsRNA (Tabara et al. 1998) revolutionised the definition of gene function in this organism and encouraged the view that the procedure would be applied readily to parasitic helminths. Here, Zhuang and Hunter (2012) review the current advances in C. elegans for RNA delivery methods, regulation of cell autonomous and systemic RNAi phenomena, and implications of enhanced RNAi mutants. They propose that these discussions, with a focus on mechanism and cross-species application, provide new perspectives for optimizing RNAi in other species.

Following the discovery that RNAi could be induced by soaking, it was not long before the first report of successful RNAi in a parasitic nematode was published with Hussein et al. (2002) reporting a large diminution in secreted AChE from Nippostrongylus brasiliensis. Soaking adult worms in dsRNA for AChE A or B, both secreted acetylcholinesterases, resulted in knockdown of the homologous enzyme activity and that of two other closely related variants of the enzyme (Hussein et al. 2002). However, transcript knockdown could not be confirmed by RTPCR. Moreover, Hussein et al. outlined in Selkirk et al. (2012) failed to observe knockdown by
\end{abstract}

RT-PCR of six other targets by soaking, these being homologues of ubiquitin, paramyosin (unc-15 in C. elegans), calponin (unc-87), protein disulphide isomerase 3 (PDI-3), phospholipase A2 (PLA2) and $\beta$-tubulin. This inconsistency of knockdown was emphasised by subsequent studies (Geldhof et al. 2007; Visser et al. 2006). Selkirk et al. (2012) discuss options which have been tested to enhance RNAi efficiency including feeding bacteria engineered to express dsRNA, methods to activate/enhance feeding, as well as the use of electroporation.

The paper by Dalzell et al. (2012) addresses many of what the authors consider to be the most important aspects of RNAi experimental design in parasitic helminths. The paper suggests ways of standardising RNAi experiments to allow meaningful comparisons to be drawn between studies in different laboratories and in different organisms. They also address issues such as parasite culture, target gene selection, the use of dsRNA or siRNA as mediators of knockdown, delivery and, most importantly, target transcript quantification and adequate controls. Finally, they propose an experiment workflow to help standardise outputs from RNAi experiments and point out some of the potential pitfalls and solutions associated with various technical aspects.

Zawadzki et al. (2012) discuss work which evaluated three different methods for introducing dsRNA into the sheep parasitic nematode Haemonchus contortus namely (1) feeding free-living stages of $H$. contortus with Escherichia coli that express dsRNA targeting the test genes; (2) electroporation of dsRNA into $H$. contortus eggs or larvae; and (3) soaking adult $H$. contortus in dsRNA. They targeted five genes that are essential in Caenorhabditis elegans (mitr-1, pat-12, vha-19, glf-1 and noah-1), orthologues of which are present and expressed in $H$. contortus, plus four genes previously tested by RNAi in $H$. contortus (ubiquitin, tubulin, paramyosin, tropomyosin). They describe reduction in transcript levels for each gene tested and, when delivery was by feeding, they noted observable changes 
in the development and viability of larvae for five of the eight genes tested, including the 'essential' genes, $H c$-pat-12, Hc-vha-19 and Hc-glf-1. Drawing together all their findings, they recommend the $E$. coli feeding method for RNAi in $H$. contortus and provide recommendations for future research directions for RNAi in this species.

Lilley et al. (2012) review impressive progress in the application of RNAi to define gene function and for the development of novel procedures for the control of root knot and cyst nematodes which affect crops. Despite the successful silencing of a range of genes, they consider that a number of limitations of RNAi in plant parasitic nematodes indicate that high-throughput screens used to identify gene function in Caenorhabditis elegans are likely to be an unrealistic expectation. Again, they highlight that differences in the methods used to deliver RNAi in vitro make it difficult to compare results between studies and thus determine those factors most crucial for achieving successful RNAi. An exciting development is the in planta delivery of RNAi to feeding nematodes which can affect the successful establishment and/or development of cyst and root-knot nematodes. This offers the prospect of transgenic delivery of dsRNA from the feeding cell to target specific, essential nematode genes as a novel means for plant parasitic nematode control. However, the available data indicate that the outcomes are variable and may differ between cyst and root-knot nematodes. In their review, the authors provide an overview of both the in vitro and in planta RNAi studies carried out to date and identify aspects of the technology that would benefit from further, systematic investigation or optimisation. They consider the potential for RNAi to be a more widely used tool than at present.

Lok (2012) provides an update on transgenesis in parasitic nematodes and examines the methods and routes of nucleic acid transfer explored to date and the application of the approach to studying the molecular, cellular or developmental biology of these organisms. Alternative modes of transgene inheritance: chromosomal or episomal are discussed as well as some problems and pitfalls associated with transgenesis in parasitic nematodes. The author considers general future applications of transgenesis in parasitic nematodes, including one suggested by studies in C. elegans (Winston et al. 2007) that could boost RNAi sensitivity in parasitic nematodes.

Advances in functional genomics and transgenesis of schistosomes are addressed by Suttiprapa et al. (2012) with a focus on approaches leading to chromosomal integration of transgenes. The retrovirus MLV and the transposon piggyBac have now both been shown to integrate reliably into the chromosomes of S. mansoni and hence both exhibit great potential as vectors to drive functional genomics for schistosomes. However, improvements are needed to establish transgenic schistosomes and protocols. An impediment has been the difficulty of delivering transgenes to the germline. Targeting integration competent transgenes to in vitro laid eggs may surmount this roadblock (Mann et al. 2010). Another option is to target the daughter sporocysts where the germ cells are comparatively massive (see Boyle et al. 2003. The authors suggest that advances in technologies such as RNAi, highthroughput insertional mutagenesis and possibly gains-of-function approaches will drive functional genomics forward quickly. They also suggest that advances in $S$. mansoni can be expected to be adapted to the other schistosomes, to the food-borne flukes such as Opisthorchis viverrini, Clonorchis sinensis and Fasciola hepatica, and other helminth parasites at large.

Beckmann and Grevelding (2012) also focus on attempts to transiently and stably transform or transfect schistosomes. Besides approaches using particle bombardment and electroporation, they discuss the approaches being used to try to achieve stable transformation using transposons and virusbased infection strategies to introduce DNA constructs expressing reporter genes into adult and larval schistosome stages. They discuss the current status of RNAi in schistosomes including establishing protocols based on soaking, lipofection, and/or electroporation. In the case of soaking, they suggest that the excretory tubules may also be involved in the uptake of dsRNAs into adult schistosomes as well as the gut. They conclude their review by taking a forward look at what might be required in the future to establish transgenesis and RNAi as practical approaches for defining gene function and, in the case of RNAi, discuss the potential impact of site of gene expression and life cycle stage targeted on a successful outcome.

MicroRNAs (miRNAs) are small, endogenous noncoding RNA molecules that post-transcriptionally regulate gene expression by targeting the $3^{\prime}$ untranslated region (3' U'TR) of messenger RNAs. First discovered in Caenorhabditis elegans, they have been shown to have important regulatory roles in many key biological processes in a variety of different organsisms. Recently, miRNAs have been identified in schistosomes and Cheng and Jin (2012) discuss the roles they may have in schistosome development and gene regulation and summarise the current status of miRNA research in schistosomes and the potential miRNAs have for the control of schistosomiasis.

\section{REFERENCES}

Beckmann, S. and Grevelding, C.G. (2012). Paving the way for transgenic schistosomes. Parasitology 139, 651-668.

Boyle, J.P., Wu, X. J., Shoemaker, C. B. and Yoshino, T.P. (2003). Using RNA interference to manipulate endogenous gene expression in 
Schistosoma mansoni sporocysts. Molecular and Biochemical Parasitology 128, 205-215.

Cheng, G. and Youxin, J. (2012). MicroRNAs: Potentially important regulators for schistosome development and therapeutic targets against schistosomiasis. Parasitology 139, 669-679.

Dalzell, J. J., Warnock, N. D., McVeigh, P., Marks, N. J., Mousley, A., Atkinson, L. and Maule, A. G. (2012). Considering RNAi experimental design in parasitic helminths. Parasitology 139, 589-604.

Hussein, A. S., Kichenin, K. and Selkirk, M.E. (2002). Suppression of secreted acetylcholinesterase expression in Nippostrongylus brasiliensis by RNA interference. Molecular and Biochemical Parasitology 122, 91-94.

Geldhof, P., Visser, A., Clark, D., Saunders, G., Britton, C., Gilleard, J., Berriman, M., Knox, D.P. (2007). RNA interference in parasitic helminthes: current situation, potential pitfalls and future prospects. Parasitology 134, 609-619.

Lilley, C. J., Davies, L. J. and Urwin, P. E. (2012). RNA interference in plant parasitic nematodes: a summary of the current status. Parasitology 139, 630-640.

Lok, J. B. (2012). Nucleic acid transfection and transgenesis in parasitic nematodes. Parasitology 139, 574-588.

Mann, V.H., Morales, M. E., Rinaldi, G. and Brindley, P. J. (2010). Culture for genetic manipulation of developmental stages of Schistosoma mansoni. Parasitology 137, 451-462.
Selkirk, M. E., Huang, S. C., Knox, D. P. and Britton, C. (2012). The development of RNA interference (RNAi) in gastrointestinal nematodes. Parasitology 139, 605-612.

Suttiprapa, S., Rinaldi, G. and Brindley, P. J. (2012). Genetic manipulation of schistosomes-progress with integration competent vectors. Parasitology 139, 641-650.

Tabara, H., Grishok, A. and Mello, C. C. (1998). RNAi in C. elegans: soaking in the genome sequence. Science 282, 430-431.

Visser, A., Geldhof, P., de Maere, V., Knox, D. P., Vercruysse, J. and Claerebout, E. (2006). Efficacy and specificity of RNA interference in larval life-stages of Ostertagia ostertagi. Parasitology 133, 777-783. Winston, W. M., Sutherlin, M., Wright, A. J., Feinberg, E. H. and Hunter, C. P. (2007). Caenorhabditis elegans SID-2 is required for environmental RNA interference. Proceedings of the National Academy for Sciences, USA 104, 10565-10570.

Zawadzki, J. L., Kotze, A. C., Fritz, J.-A., Johnson, N. M., Hemsworth, J. E., Hines, B. M. and Behm, C. A. (2012). Silencing of essential genes by RNA interference in Haemonchus contortus. Parasitology 139, 613-629.

Zhuang, J. J. and Hunter, C. P. (2012). RNA interference in Caenorhabditis elegans: Uptake, mechanism, and regulation. Parasitology 139, 560-573.

DAVE KNOX

Fanuary 2012 